Mater. Res. Soc. Symp. Proc. Vol. 1621 ㄷ 2014 Materials Research Society

DOI: 10.1557/opl.2014.67

\title{
Size Also Matters in Biodegradable Composite Microfiber Reinforced by Chitosan Nanofibers
}

\author{
Elisabete D. Pinho ${ }^{1,2}$, Albino Martins ${ }^{1,2}$, José V. Araújo ${ }^{1,2}$, Rui L. Reis ${ }^{1,2}$ and Nuno M. Neves ${ }^{1,2}$ \\ ${ }^{1}$ 3B's Research Group - Biomaterials, Biodegradables and Biomimetics. Department of \\ Polymer Engineering, University of Minho; Headquarters of the European Institute of Excellence \\ on Tissue Engineering and Regenerative Medicine. AvePark, Zona Industrial da Gandra, S. \\ Cláudio do Barco 4806-909 Caldas das Taipas, Guimarães, Portugal. \\ ${ }^{2}$ ICVS/3B's, PT Government Associate Laboratory, Braga/Guimarães, Portugal.
}

\begin{abstract}
Pioneer works on nanocomposites were focused in carbon nanofibers or nanotubes dispersed in epoxy matrix, a viscous liquid facilitating the compounding stage. The interest in developing new composites aimed for biomedical applications led us to design new nanocomposites based in biodegradable polymers with demonstrated biological performance.

We report herein the development of micro-nano composites by extruding poly(butylene succinate) (PBS) microfibers with two different diameters, 200 and $500 \mu \mathrm{m}$, reinforced with electrospun chitosan nanofibers. Analysis of the microfibers showed high levels of alignment of the reinforcing phase and excellent distribution of the nanofibers in the composite. Its geometry facilitates the development of orthotropy, maximizing the reinforcement in the axial fiber main axis.

The biodegradable microfiber composites show an outstanding improvement of mechanical properties and of the kinetics of biodegradation, with very small fractions ( 0.05 and 0.1 wt.\%) of electrospun chitosan nanofibers reinforcement. The high surface area-to-volume ratio of electrospun nanofibers combined with the increased water uptake capability of chitosan justify the accelerated kinetics of biodegradation of the composite as compared with the unfilled synthetic polymer.
\end{abstract}

\section{INTRODUCTION}

Nanocomposites are a class of advanced materials consisting in a reinforcing phase with some dimensions in the nanometer size range dispersed in a continuous matrix. Usually the nanometer dimension range is defined between 1 and $100 \mathrm{~nm}$. This requirement is not consensually accepted, depending on the characteristics of the nanoentities of interest and on the field of application [1]. The nanocomposites may exhibit enhanced properties such as the elastic modulus, strength, heat resistance, barrier properties, transparency or biodegradability. The superior properties of nanocomposites have potential interest for many applications in electronic, automotive or biomedical industries [2].

Fibrous composites allow easier structuring of reinforcements being more effective than bulk materials. Its geometry facilitates the development of orthotropy, maximizing the reinforcement in the fiber main axis. The production of composite fibers by melt processing 
using extrusion is a very well established technology [3,4]. The melt flow dynamics in the extrusion of fibers facilitate the alignment of reinforcements within the composite and the reinforcing efficiency.

The maximum reinforcement efficiency is obtained when the nanofibers are uniformly dispersed and individually coated with the continuous matrix of the composite [3]. A good fiber dispersion facilitates the load transfer and development of uniform stress distribution and minimizes the probability of development of structural defects or voids acting as stressconcentration points. The alignment also allows maximizing strength and stiffness in particular directions [5]. Composites intended for biomedical applications such as wound care, drug delivery, medical devices or scaffolds for tissue engineering have the critical requirement of the biocompatibility. The biodegradability is also very important if those materials are developed for temporary devices such as resorbable sutures or tissue engineering scaffolds $[4,6]$.

The pioneer work on nanofiber composites was focused in carbon nanofibers or nanotubes [3]. Those nano-reinforced composites may be produced by polymer processing technology using solvents and surfactants to avoid nanofiber agglomeration. Electrospun polymer nanofiber meshes were explored as composite reinforcements in a small number of studies $[4,7]$. Those reports were mainly centered in the reinforcing effect of electrospun nanofibers dispersed in an epoxy matrix [7].

Electrospinning is an efficient technique for the production of nanofibers with diameters in the submicrometer range $[8,9]$. The increased surface area-to-volume ratio as a consequence of the submicron diameter is one of the most interesting properties of electrospun fibers $[10,11]$. The nanoscale size of the biodegradable fibers may also offer advantages in terms of inducing specific degradation kinetics. In this study we successfully produced and stabilized electrospun nanofibers from chitosan. Chitosan is an alkaline deacetylated derivative of chitin that may be obtained from the shells of arthropods or cephalopods. This material in combination with various biodegradable polyesters has already shown a good combination of properties for different biomedical applications [12-15]. The poly(butylene succinate) (PBS) matrix showed previously stronger biological performance in combination with chitosan, being thus selected for the present study. The biodegradable aliphatic polyester PBS, presenting a hydrophobic character, shows slow degradation kinetics. Chitosan is rich in polar groups $\left(-\mathrm{OH}\right.$ and $\left.-\mathrm{NH}_{2}\right)$ and is very hydrophilic. Its presence as reinforcement in the microfiber composite increases the final hydrophilicity of the composite, typically being determined by the wettability of the continuous phase [16]. The high surface area-to-volume ratio combined with the increase in the water uptake capability of polymer reinforced composite fiber is intended to accelerate the kinetics of biodegradation of the composite.

Fibers of poly(butylene succinate) (PBS) with and without the nanofiber reinforcement were produced in two diameters $(200$ and $500 \mu \mathrm{m})$ to evaluate the efficiency of reinforcement in those two fiber dimensions. The effect of the chitosan nanofiber meshes (Cht NFM) reinforcements concentration was evaluated by compounding the composites with $0.05 \%$ and $0.1 \%$ by weight. The analysis of the composite fibers included the study of there morphology, mechanical properties and the kinetics of degradation, after various periods of immersion in an isotonic saline solution. We also characterized the thermal properties of the composite by thermogravimetrical analysis and calorimetry, and the composites chemical composition by Attenuated Total Reflection-Fourier Transform InfraRed (ATR-FTIR). 


\section{EXPERIMENTAL DETAILS}

\section{$\underline{\text { Materials }}$}

Poly(butylene succinate) (PBS) Bionolle ${ }^{\mathrm{TM}} 1050$ was supplied by Showa Highpolymer Co. Ltd., Tokyo, Japan. Trifluoroacetic acid (TFA) was obtained from Sigma and Dichloromethane (DCM) was supplied by Aldrich. Ammonia $7 \mathrm{~N}$ solution in methyl alcohol was purchased from Aldrich and Methanol was obtained from Fluka.

\section{Production of Chitosan Nanofiber Meshes (Cht NFM)}

The electrospinning processing and the subsequent neutralization process were conduct according to the protocol described elsewhere [4]. Briefly, a polymeric solution of $4 \%(\mathrm{w} / \mathrm{v})$ chitosan $(\mathrm{Mv}=417 \mathrm{kDa} ; \mathrm{DD}=88 \%)$ was prepared using a solvent mixture of TFA and DCM (7:3 ratio). Electrospinning of this polymeric solution was achieved by establishing a electric field of $16-18 \mathrm{kV}$, a needle-to-ground collector distance of $12-14 \mathrm{~cm}$ and a flow rate of $0.6-0.8 \mathrm{~mL} / \mathrm{h}$. Neutralization of Cht NFM was performed by immersing them in a solution containing concentrated ammonium and methanol.

\section{Production of Microfibers by Melt Extrusion}

Three different types of compositions were produced in this study: i) PBS fiber; ii) Fiber composed by PBS ( $99.95 \%$ by weight) reinforced with $0.05 \%$ by weight of Cht NFM; iii) Fiber composed by PBS ( $99.9 \%$ by weight) reinforced with $0.1 \%$ by weight of Cht NFM. The processing conditions were optimised to obtain a stable extrusion processing. The processing conditions were kept constant for all compositions to maintain the same thermal history in all compositions. The melt temperature was set at $115^{\circ} \mathrm{C}$ and the screw rotation speed at 40 r.p.m. The die diameters used were either 0.2 or $0.5 \mathrm{~mm}$ for each composition.

\section{$\underline{\text { Morphological Analysis }}$}

The morphology of the microfiber composites (with and without nanofiber mesh reinforcements) was analysed by scanning electron microscopy (SEM) (model S360, Leica Cambridge) after being sputter coated with gold. To analyse the morphology, distribution and structure of the chitosan nanofibers inside of the composite microfibers, a cryogenic fracture of the fiber or a partial dissolution of the polyester matrix with dichloromethane was performed.

\section{Surface Chemistry Characterization}

The chemical characterization of the surfaces was firstly performed by Attenuated Total Reflection-Fourier Transform InfraRed (ATR-FTIR). The FTIR spectra was recorded on an IRPrestige 21 FTIR spectrophotometer (Shimadzu, Japan) with a resolution of $4 \mathrm{~cm}^{-1}$ and averaged over 36 scans. 


\section{Differential scanning calorimetry (DSC)}

The measurements were carried out using a Perkin Elmer DSC-7 instrument under a dynamic nitrogen atmosphere. The samples were heated to $30^{\circ} \mathrm{C}$ to $150{ }^{\circ} \mathrm{C}$ by $10{ }^{\circ} \mathrm{C} / \mathrm{min}$. The mass of the samples was around $5 \mathrm{mg}$. The calculations were making by Avrami model. The degree of crystallinity of the PBS component was calculated from the known theoretical value of $\Delta H_{\mathrm{f}}$ can be compared with $110.3 \mathrm{~J} / \mathrm{g}$, corresponding to $100 \%$ crystalline PBS calculated on the basis of the group contribution method proposed by Van Krevelen [17].

\section{$\underline{\text { Thermogravimetric analysis (TGA) }}$}

A thermogravimetric analyzer (Q500, TA Instruments, New Castle-Delaware, USA) was used to investigate the effect of Cht NFM processing on the polymer. PBS fibers (without reinforcement) and PBS fibers reinforced by Cht NFM were analyzed in closed Platinum cups in a temperature range of $30-1000{ }^{\circ} \mathrm{C}$. The heating rate established was $10{ }^{\circ} \mathrm{C} / \mathrm{min}$. All the experiments were carried out in a nitrogen atmosphere (flow rate $60 \mathrm{~cm}^{3} / \mathrm{min}$ ).

\section{$\underline{\text { Mechanical Tests }}$}

The reinforced composite fibers were cut in $10 \mathrm{~mm}$ long fiber specimens with diameter of approximately 200 and $500 \mu \mathrm{m}$. The tensile tests were performed at room temperature in a Universal Mechanical Test Machine (Instron 4505) using a load cell of $1 \mathrm{KN}$ and a crosshead speed of $5 \mathrm{~mm} / \mathrm{min}$. A minimum of six specimens were tested in each sample (the values reported are the average of those results).

\section{Swelling and Degradation-Related Tests}

The hydration degree and degradation behaviour of the particulate microfiber compositions were assessed over a period of 30 days. Five specimens of each sample (previously weighed) were immersed in an isotonic saline solution (ISS: $0.154 \mathrm{M} \mathrm{NaCl}$ aqueous solution, $\mathrm{pH}$ $=7.4$ ) during $1,3,7,14$ and 30 days.

\section{RESULTS \& DISCUSSION}

The morphology of the microfiber composites (with and without nanofiber mesh reinforcements) was analyzed by scanning electron microscopy (SEM) and are showed in Figure $1 \mathrm{a}$ ) and b). The fiber, shown in Figure $1 \mathrm{a}$ ), was produced with a die diameter of $500 \mu \mathrm{m}$ (resulting in a fiber diameter of approximately $460 \mu \mathrm{m}$ ). In Figure $1 \mathrm{~b}$ ) it is shown a fiber produced with a die diameter of $200 \mu \mathrm{m}$ (fiber diameter of approximately $210 \mu \mathrm{m}$ ). The fibers are characterized by a regular and smooth surface confirming the effectiveness of the extrusion processing.

The chitosan nanofiber mesh obtained by electrospinning is soluble in water and is not structurally stable. Its use in biomedical applications implies a neutralization process to chemically stabilize the chitosan but preserving the morphology of the nanofiber mesh. In a previous work we reported that electrospun Cht NFM, after the neutralizing alkaline treatment, 


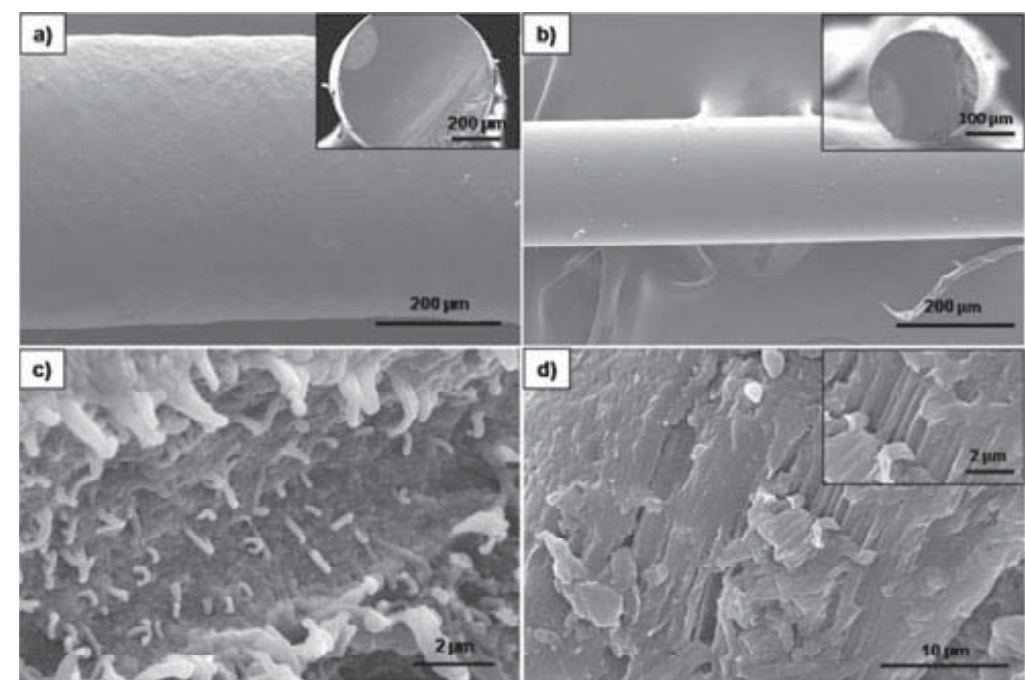

Figure 1. SEM micrographs of: a) fiber with $460 \mu \mathrm{m}$ of diameter (fiber500), b) fiber with 210 $\mu \mathrm{m}$ of diameter (fiber200), c) cross-section of PBS fiber reinforced by Cht NFM and d) longitudinal section.

successfully maintained the electrospun nanofiber morphology [4]. The fibers have diameters ranging from $65 \mathrm{~nm}$ to $6 \mu \mathrm{m}$, exhibiting a regular and smooth surface topography without the presence of beads which is commonly referred as a problem of electrospun nanofiber meshes [9]. The mesh-like structure has interconnected pores and a non-woven, random distribution. Those observations confirm the efficacy of the optimized conditions used both in the electrospinning process and in the subsequent neutralization treatment.

As expected, the Cht nanofibers are present underneath the fiber surface in the inner core of the fibers (being only exposed after cryogenic fracture of the fiber sample). The reinforced composite fiber combines the micrometer size of the extruded fiber with the nanoscale of the reinforcement. The biodegradable PBS matrix reinforced by Cht NFM shows the nanofiber mesh highly aligned after the processing. This alignment is not present before the extrusion process and is probably generated by the hydrodynamics of the flowing melt during the extrusion process [18]. During polymer melt extrusion a stress field develops caused by the viscous nature of the melt. The stresses acting in the melt are predominantly shear stresses that are known to cause the alignment of fibers in the main flow direction. This effect can be observed both in cross section (Figure $1 \mathrm{c}$ ) and also in longitudinal section (Figure $1 \mathrm{~d}$ ) micrographs. The micrographs show the alignment of the nanofibers with the main axis of the fiber and the intimate coverage of the $\mathrm{Cht}$ NFM by the continuous phase provided by the extrusion process. The distribution of the nanofibers and the adhesion matrix/nanofibers (Figure $1 \mathrm{c}$ and d) shows an excellent interface without visible voids or defects. The high alignment of the Cht NFM in the composite suggests that significant anisotropy of mechanical properties may exist [3-5].

The surface chemistry of the fiber composites, the PBS fibers and the chitosan nanofiber meshes were all analyzed by FTIR-ATR. The spectra of the different fiber materials and composites are shown in Figure 2 a). The characteristic absorption bands were assigned to the 
a)

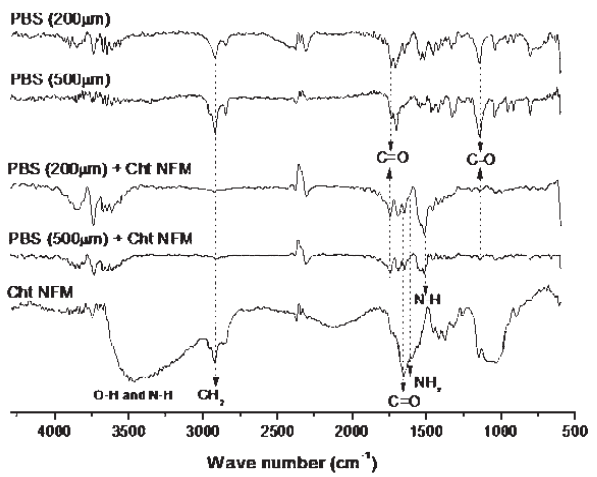

c)

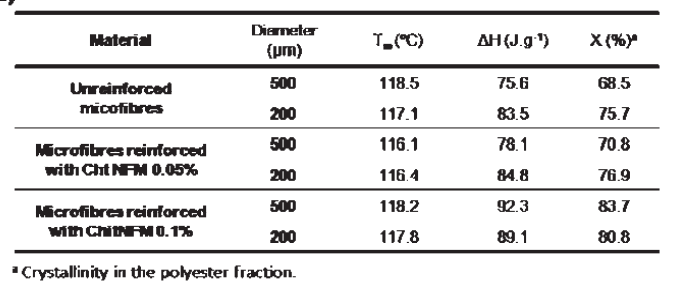

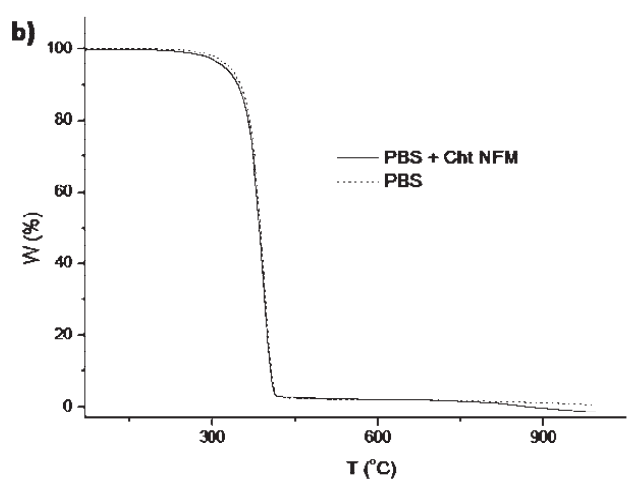

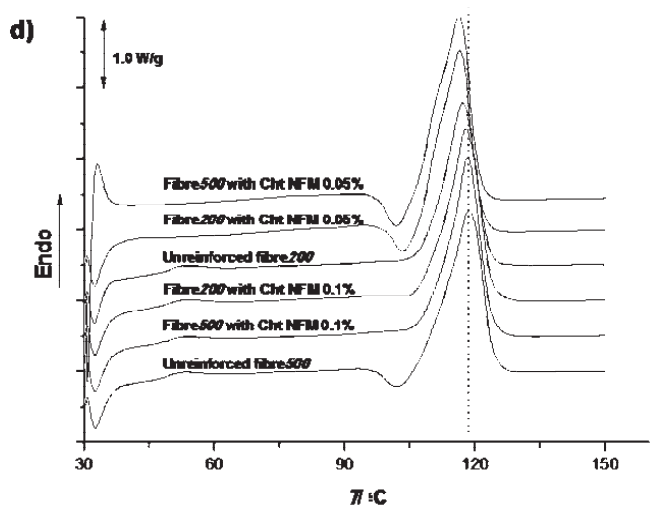

Figure 2. Chemical and thermal analysis: a) FTIR-ATR for the unreinforced and reinforced PBS fibers by Cht NFM; b) Representative thermogravimetric curves of the processing fibers; $\mathbf{c})$ Melting temperature and heat of fusion of the different samples analyzed by DSC, and the corresponding crystallinity degree within the synthetic polymer component; d) DSC thermograms for unreinforced fibers and fibers reinforced by Cht NFM.

components of the reinforced composite fiber. The stretching bands corresponding to $\mathrm{O}-\mathrm{H}$ and $\mathrm{N}-\mathrm{H}\left(3480-3080 \mathrm{~cm}^{-1}\right), \mathrm{CH}_{2}\left(2960-2560 \mathrm{~cm}^{-1}\right), \mathrm{C}=\mathrm{O}\left(1648 \mathrm{~cm}^{-1}\right), \mathrm{NH}_{2}\left(1612 \mathrm{~cm}^{-1}\right)$ and $\mathrm{N}-\mathrm{H}$ $\left(1509 \mathrm{~cm}^{-1}\right)$ are all assigned to chitosan. The stretching bands corresponding to $\mathrm{C}=\mathrm{O}\left(1741 \mathrm{~cm}^{-1}\right)$, $\mathrm{C}-\mathrm{O}\left(1140 \mathrm{~cm}^{-1}\right)$ and C-H $\left(1147-1263 \mathrm{~cm}^{-1}\right)$ are assigned to PBS [16]. As was expected, the spectrum of the reinforced composite fiber presented the main chemical groups of the Cht NFM. In some cases the lower intensity bands of chitosan were overlapped by the much sharper and higher intensity bands of PBS and, then, are more difficult to be detected in the reinforced composite fibers.

Figure $2 \mathrm{~b}$ ) shows the TGA curves for the materials subjected to extrusion processing. TGA was used in this study to observe the effect of Cht NFM on the thermogravimetrical properties of the composite. It is important to verify if the processing temperatures used in extrusion cause any significant thermogravimetrical variation. The small mass loss observed at the extrusion temperature is probably due to loss of moisture or minor volatile reagents present in the material as processing additives. The presence of $0.1 \%$ of Cht NFM in the fiber composite does not affect the pattern of thermal degradation. TGA data shows that the thermal degradation initiates at approximately $230{ }^{\circ} \mathrm{C}$. Most of the material undergoes volatilization at $416^{\circ} \mathrm{C}$. Both 
profiles showed similar thermal degradation. The infrared spectroscopy results together with the TGA analysis allow confirming the absence of thermal degradation of chitosan in the produced reinforced composite fibers.

The results from differential scanning calorimetry (DSC) experiments for unreinforced fibers and fibers reinforced by Cht NFM are shown in Figure 2 d). The melting of the synthetic phase was clearly detected in all the fibers (endothermic peak). Figure $2 \mathrm{c}$ ) summarizes the thermal characteristics of the fibers. It is noted the increase of the $\Delta H$ with increasing Cht NFM content in the PBS fibers. The degree of crystallinity of the PBS component was calculated from the estimated theoretical value of $\Delta H$ for $100 \%$ crystalline PBS, having a value $110.3 \mathrm{~J} / \mathrm{g}$. This value was calculated on the basis of the group contribution method proposed by Van Krevelen [17]. PBS-based materials show some tendency for the depression of Tm with the introduction of the Cht NFM reinforcement, except for the fiber500 and fiber 200 reinforced with $0.1 \%$ of Cht NFM. This trend may be caused by the chitosan fibers acting as a nucleating agent and lowering the size of the crystalline structures. This phenomenon could also explain the increase in the level of crystallinity observed in the reinforced composite fibers. This possible effect of Cht NFM over the nucleation and development of crystallinity of the PBS matrix may also influence the mechanical properties of the composite, eventually by creating a strong interface between the matrix and the reinforcement $[3,5]$.

The tensile modulus, tensile stress and tensile strain are all shown in Table I. A value of $31 \mathrm{MPa}$ was obtained for the tensile yield stress (strength) of the unreinforced fiber500 and tensile strain of $12 \%$. The unreinforced fiber 200 presents much higher values of both the tensile stress and strain, $75 \mathrm{MPa}$ and $46 \%$ respectively. The fiber 500 without reinforcement has tensile modulus of $329 \mathrm{MPa}$ and the fiber200 shows much higher tensile modulus ( $950 \mathrm{MPa})$. This result may be explained by the increase in molecular orientation experienced by PBS when extruded into a thinner fiber. The mechanical properties of the fibers 200 can be also interpreted together with DSC results (Figure $2 \mathrm{c}$ ). DSC results show that the unreinforced fiber200 presents a higher crystallinity that directly influences the mechanical properties. A higher degree of crystallization corresponds typically to higher modulus and tensile strength. However, the differences in crystallinity observed are not sufficient to explain the threefold increase in tensile modulus.

Despite the modest fraction of Cht NFM reinforcement, the reinforced fibers show both tensile modulus and tensile stress significantly enhanced. The maximum tensile strength was obtained for fiber 200 reinforced with $0.05 \%$ of Cht NFM having a value of $154 \mathrm{MPa}$. This value is much higher than that obtained for fiber 500 with $0.1 \%$ of Cht NFM. The tensile strain properties of fibers reinforced by Cht NFM shows a reduction of the ultimate tensile strain with increasing Cht NFM content. This result is also an indication of a strong reinforcement of the fibers by the presence of the Cht NFM, having associated a typical decrease in tensile strain [19]. The PBS fiber reinforced by Cht NFM showed a maximum increase in the tensile modulus in the case of fiber 200 with $0.1 \%$ of Cht NFM. A value of the modulus of $2017 \mathrm{MPa}$ was obtained, representing the double of that obtained for unreinforced fiber200 (949.8 MPa). The composite fiber 500 with $0.05 \%$ of Cht NFM showed a tensile modulus of $553.2 \mathrm{MPa}, 70 \%$ larger than the unreinforced fiber500 (329.3 MPa). The reinforcement by Cht NFM is much less effective in fiber500 than in fiber200. The reinforcement resulting from the orientation along the main axis of the fiber micro/nanocomposites probably is probably responsible for those differences. 
Table I. Tensile properties of fiber 500 and fiber 200 reinforced or not with Cht NFM, obtained from the tensile curves, before and after being subjected to swelling and degradation tests.

\begin{tabular}{|c|c|c|c|c|c|}
\hline Materials & Immersion Time [days] & Diameter $[\mu \mathrm{m}]$ & Tensile Strength [MPa] & Tensile Modulus [MPa] & Tensile Strain [\%] \\
\hline \multirow{12}{*}{$\begin{array}{l}\text { Unreinforced } \\
\text { microfibers }\end{array}$} & \multirow{2}{*}{0} & 500 & $30.6 \pm 7.2$ & $329.3 \pm 36.9$ & $11.6 \pm 2.3$ \\
\hline & & 200 & $74.5 \pm 11.1$ & $949.8 \pm 197.3$ & $46.0_{ \pm} 10.2$ \\
\hline & \multirow{2}{*}{1} & 500 & $19.3 \pm 6.3$ & $322.1 \pm 87.6$ & $8.8 \pm 1.2$ \\
\hline & & 200 & $46.9 \pm 5.9$ & $597.8 \pm 62.2$ & $41.4 \pm 16.7$ \\
\hline & \multirow{2}{*}{3} & 500 & $18.2 \pm 4.9$ & $308.1 \pm 8.4$ & $8.0 \pm 1.5$ \\
\hline & & 200 & $51.1 \pm 12.8$ & $594.3 \pm 73.6$ & $14.3 \pm 4.3$ \\
\hline & \multirow{2}{*}{7} & 500 & $17.7 \pm 6.4$ & $302.8 \pm 64.3$ & $7.3 \pm 0.8$ \\
\hline & & 200 & $35.6 \pm 10.3$ & $654.7 \pm 128.6$ & $15.9 \pm 11.6$ \\
\hline & \multirow{2}{*}{14} & 500 & $11.2 \pm 3.4$ & $140.8 \pm 54.6$ & $8.8 \pm 1.5$ \\
\hline & & 200 & $28.3 \pm 7.2$ & $540.2 \pm 62.9$ & $15.0 \pm 7.5$ \\
\hline & \multirow{2}{*}{30} & 500 & $5.6 \pm 1.6$ & $129.8 \pm 61.9$ & $4.6 \pm 1.0$ \\
\hline & & 200 & $7.1 \pm 1.5$ & $425.0 \pm 136.0$ & $5.8 \pm 2.5$ \\
\hline \multirow{12}{*}{$\begin{array}{l}\text { Microfibers reinforced } \\
\text { with Cht NFM } 0.05 \%\end{array}$} & \multirow{2}{*}{0} & 500 & $35.4 \pm 6.4$ & $553.2 \pm 48.4$ & $10.7 \pm 0.9$ \\
\hline & & 200 & $154.0_{ \pm} 34.2$ & $1783.5 \pm 238.5$ & $29.2 \pm 10.2$ \\
\hline & \multirow{2}{*}{1} & 500 & $32.6 \pm 9.5$ & $513.6 \pm 77.3$ & $11.2 \pm 2.3$ \\
\hline & & 200 & $104.3 \pm 12.8$ & $1085.5 \pm 330.6$ & $32.5 \pm 21.7$ \\
\hline & \multirow{2}{*}{3} & 500 & $25.5 \pm 2.6$ & $492.7 \pm 55.1$ & $7.6 \pm 2.5$ \\
\hline & & 200 & $55.5 \pm 11.8$ & $858.7 \pm 137.0$ & $12.8 \pm 4.4$ \\
\hline & \multirow{2}{*}{7} & 500 & $24.9 \pm 6.9$ & $475.9 \pm 34.3$ & $8.9 \pm 3.5$ \\
\hline & & 200 & $42.7 \pm 10.2$ & $1127.7 \pm 10.2$ & $7.5 \pm 1.8$ \\
\hline & \multirow{2}{*}{14} & 500 & $18.3 \pm 1.2$ & $446.3 \pm 113.3$ & $7.4 \pm 1.9$ \\
\hline & & 200 & $19.6 \pm 7.7$ & $736.4 \pm 129.0$ & $8.1 \pm 6.4$ \\
\hline & \multirow{2}{*}{30} & 500 & $11.7 \pm 2.8$ & $395.9 \pm 33.7$ & $4.1 \pm 1.1$ \\
\hline & & 200 & $14.0 \pm 4.5$ & $629.6 \pm 134.0$ & $9.2 \pm 5.9$ \\
\hline \multirow{12}{*}{$\begin{array}{l}\text { Microfibers reinforced } \\
\text { with Cht NFM } 0.1 \%\end{array}$} & \multirow{2}{*}{0} & 500 & $39.0 \pm 8.3$ & $543.4 \pm 69.0$ & $17.3 \pm 4.5$ \\
\hline & & 200 & $136.7 \pm 23.9$ & $2016.7 \pm 131.2$ & $19.3 \pm 10.7$ \\
\hline & \multirow{2}{*}{1} & 500 & $28.7 \pm 2.5$ & $572.6 \pm 43.1$ & $9.8 \pm 2.9$ \\
\hline & & 200 & $72.1 \pm 22.7$ & $1046.1 \pm 187.8$ & $19.9 \pm 8.9$ \\
\hline & \multirow{2}{*}{3} & 500 & $15.3 \pm 6.3$ & $459.2 \pm 39.0$ & $7.2 \pm 3.8$ \\
\hline & & 200 & $38.1 \pm 8.2$ & $716.7 \pm 170.8$ & $11.3 \pm 5.1$ \\
\hline & \multirow{2}{*}{7} & 500 & $19.3 \pm 4.0$ & $491.7 \pm 90.9$ & $6.7 \pm 0.7$ \\
\hline & & 200 & $33.8 \pm 14.2$ & $931.4 \pm 158.4$ & $13.9 \pm 8.8$ \\
\hline & \multirow{2}{*}{14} & 500 & $8.7 \pm 4.1$ & $437.3 \pm 84.7$ & $6.4 \pm 2.5$ \\
\hline & & 200 & $29.9 \pm 10.9$ & $802.4 \pm 167.2$ & $10.0 \pm 8.1$ \\
\hline & \multirow{2}{*}{30} & 500 & $7.5 \pm 3.0$ & $326.5 \pm 87.6$ & $7.5 \pm 2.5$ \\
\hline & & 200 & $15.8 \pm 4.9$ & $658.0 \pm 107.9$ & $4.5 \pm 1.4$ \\
\hline
\end{tabular}

Continuous and highly aligned reinforcements in composites are more effective in achieving higher mechanical properties and also higher anisotropy (or orthotropy) [20]. Additionally, the nanofibers being longer and well oriented provide more surface area to develop a good interface with the matrix. As a result, not only the stiffness but also the tensile strength of the fibers reinforced by Cht NFM may be increased (Table I). The interface properties are critical to ensure the load transfer process and increase the reinforcing efficiency. The submicron diameters of the Cht NFM also help in avoiding the formation of defects that could limit the tensile strength. 
Biomaterials developed to operate in immersion conditions should be evaluated in terms of swelling and hydrophilic/hydrophobic character. The rate of water uptake is related to various physical and chemical properties such as hydrophilicity, crystallinity or surface area [21]. Figure 3 a) and b) show the water absorption profiles of Cht NFM, fiber500 and fiber200 with and without reinforcement. Statistically significant differences in water uptake capacity are observed depending on the composition. The different diameters of the fibers also influence the water absorption being explained by the diffusion length. The fiber 500 have maximum water uptake of $2.4 \%$ and the fiber 200 have $5.4 \%$ of water uptake capacity. The smaller fiber diameter has also higher surface area per unit of volume, which further increments the water uptake ability. Figure 3 a) shows that the fiber 500 and the fiber 500 with $0.1 \%$ of Cht NFM reached a maximum of $8 \%$ in water uptake at day 14 . In Figure 3 b) the fiber 200 reinforced with $0.1 \%$ of Cht NFM increased the water uptake to $13.3 \%$, reaching also a maximum at day 14 . The low amount of Cht NFM used in the composites shows an outstanding increment in water absorption. The results are probably related with the high surface area of the nanofibers, facilitating the intake of water, and the properties of chitosan, a highly hydrophilic natural polymer (Cht NFM water uptake is $300 \%$ ). The limit value of the water uptake may be regarded as a result of two
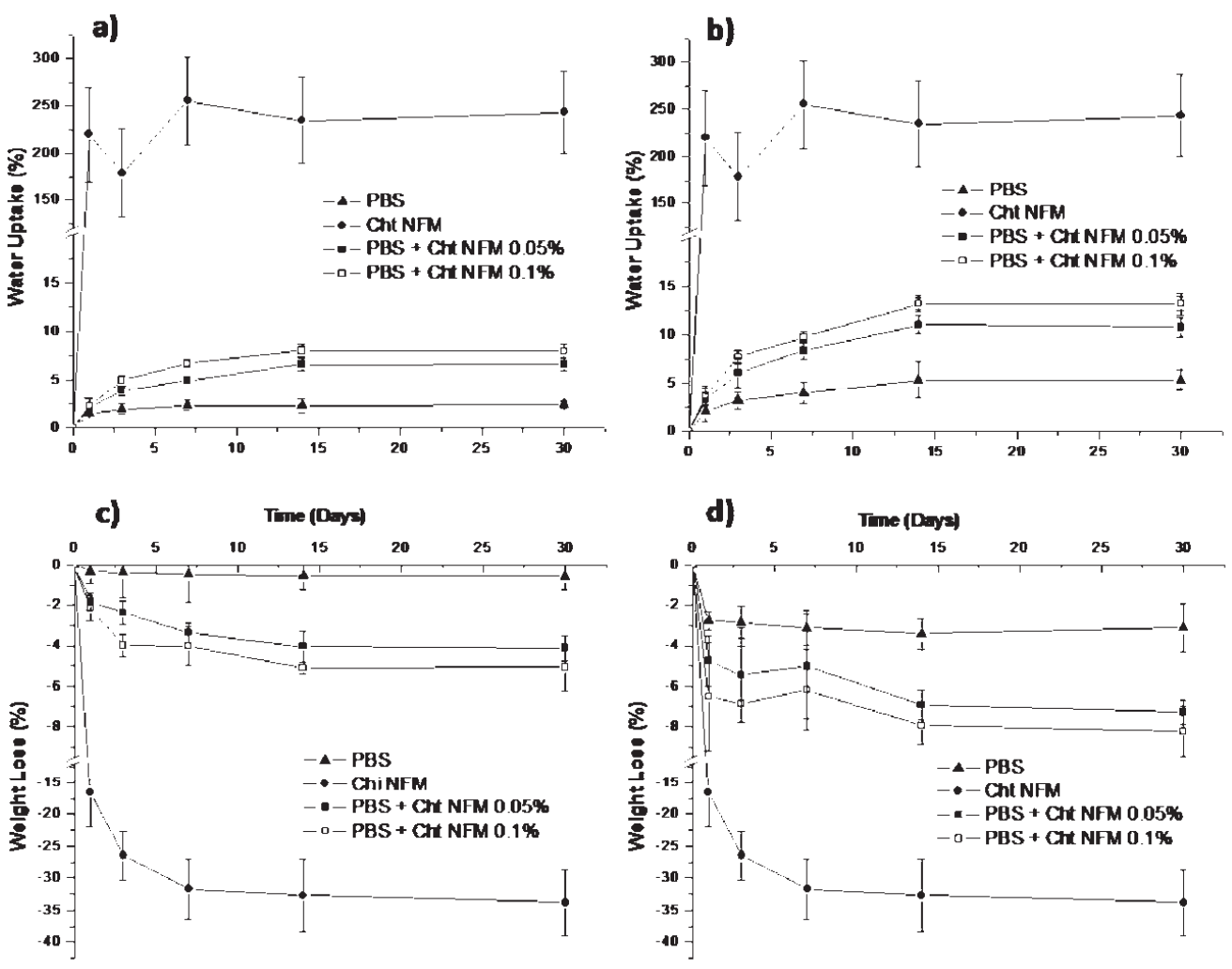

Figure 3. a) and b) Water uptake percentage along experimental time course for fiber500 and fiber200, respectively, with or without reinforcement and Cht NFM; c) and d) represents the weight loss percentage along different degradation times of fibers reinforced by Cht NFM, chitosan nanofiber meshes and unreinforced fibers. 
complementary phenomena: the intrinsic water uptake capability of the material; and the degradation history. Those phenomena are somewhat inter-related, because the water absorption usually accelerates the degradation process, particularly in materials that are sensitive and undergo hydrolysis [22].

Figure $3 \mathrm{c}$ ) and d) show the weight loss results of the fibers with and without Cht NFM reinforcements. The weight loss of the unreinforced fiber 500 and fiber 200 reached a maximum value of $0.5 \%$ and $3.4 \%$, respectively, after being subjected to 30 days of immersion. This shows that the degradation is still slow in the composite, but faster than that of PBS.

The mechanical properties of the fibers after the degradation tests decrease, as expected (Table I). The fibers reinforced by Cht NFM show decrease of modulus with time of immersion. The tensile modulus of fiber 500 reinforced with $0.05 \%$ of Cht NFM after 30 days of immersion was higher than that of unreinforced fibers before being subject to immersion tests (the initial tensile modulus). This result (Table I) may be also considered a remarkable property of these reinforced composite fibers.

Composites reinforced with $0.1 \%$ of Cht NFM (fiber500 and fiber 200 ) do not show dramatic differences except in the kinetics of degradation of fiber 200 probably caused by the larger surface area facilitating the diffusion. The variation of tensile strength during the periods of immersion was not as significant as the variation in the tensile modulus. The fibers reinforced by Cht NFM show comparable values of the ultimate tensile strain when compared with the unreinforced ones after 30 days of immersion. The mechanical properties clearly increased by the composition with Cht NFM, as well as the degradation kinetics previously reported.

\section{CONCLUSIONS}

Novel micro-nano composites were developed by producing biodegradable polymeric fibers reinforced by electrospun polysaccharide nanofibers. The nanofibers, although being initially obtained in randomly aligned meshes, have a well-aligned morphology inside the composite fibers. The fibers reinforced by nanofibers show a considerable alignment of the Cht NFM along its longitudinal main axis. This alignment is caused by the dynamics of the flowing melt during extrusion and by the stress fields. The Cht NFM reinforcement in the fibers also increased the water uptake. The weight loss was increased, indicating that the kinetics of biodegradation was significantly accelerated by the presence of the nanofiber reinforcement. Also the diameter causes variation in the kinetics of biodegradation being faster in the thinner fibers.

The elastic modulus of the fiber composite was significantly enhanced, even with very modest contents of nanofiber reinforcements. The tensile strength was marginally improved and the tensile strain is reduced considerably as is typical for fiber-reinforced composites. Thinner fibers present the same trends but higher efficiency of reinforcement. The increase of the crystallinity with the introduction of Cht NFM in the fibers also positively contributes for enhancing the mechanical properties of the fiber composites. The new nanofiber composite shows an outstanding improvement of mechanical properties and kinetics of biodegradation with very modest fractions of reinforcement and represents a new strategy to design materials with tailored properties and enhanced performance for many applications. 


\section{ACKNOWLEDGMENTS}

This work was partially supported by the OsteoGraphy (PTDC/EME-MFE/2008) and MaxBone (PTDC/SAU-ENB/115179/2009) projects, financed by the Portuguese Foundation for Science and Technology (FCT).

\section{REFERENCES}

1. E. Stodolak, C. Paluszkiewicz, M. Bogun and M. Blazewicz, J. Mol. Struc., 924-926, 208 (2009).

2. Q. Y. Soundararajah, B. S. B. Karunaratne and R. M. G. Rajapakse, Mater. Chem. Phys. 113, 850 (2009).

3. J. N. Coleman, U. Khan and Y. K. Gun'ko, Adv. Mater. 18, 689 (2006).

4. E. D. Pinho, A. Martins, J. V. Araújo, R. L. Reis and N. M. Neves, Acta Biomater. 5, 1104 (2009).

5. L.-Q. Liu, D. Tasis, M. Prato and H. D. Wagner, Adv. Mater. 19, 1228 (2007).

6. L. Y. Yeo and J. R. Friend, J. Exp. Nanosci. 1, 177 (2006).

7. Z.-M. Huang, Y.-Z. Zhang, M. Kotaki and S. Ramakrishna, Compos. Sci. Technol. 63, 2223 (2003).

8. J. Venugopal, P. Vadgama, T. S. S. Kumar and S. Ramakrishna, Nanotechnology 18, 511 (2007).

9. A. Martins, R. L. Reis and N. M. Neves, Int. Mater. Rev. 53, 257 (2008).

10. T. Lin, H. Wang and X. Wang. Adv. Mater. 17, 2699 (2005).

11. A. Martins, E. D. Pinho, S. Faria, I. Pashkuleva, A. P. Marques, R. L. Reis and N. M. Neves, Small 5, 1195 (2009).

12. V. M. Correlo, L. F. Boesel, M. Bhattacharya, J. F. Mano, N. M. Neves and R. L. Reis, Macrom. Mater. Eng. 290, 1157 (2005).

13. V. M. Correlo, E. D. Pinho, I. Pashkuleva, M. Bhattacharya, N. M. Neves and R. L. Reis, Macromol. Biosci. 7, 354 (2007).

14. A. R. Costa-Pinto, A. J. Salgado, V. M. Correlo, P. C. Sol, M. Bhattacharya, P. Charbord, R. L. Reis and N. M. Neves, Tissue Eng. Part A 14, 1049 (2008).

15. J. T. Oliveira, V. M. Correlo, P. C. Sol, A. R. Costa-Pinto, P. B. Malafaya, A. J. Salgado, M. Bhattacharya, P. Charbord, N. M. Neves and R. L. Reis, Tissue Eng. Part A 14, 1651 (2008).

16. D. F. Coutinho, I. Pashkuleva, C. M. Alves, A. P. Marques, N. M. Neves and R. L. Reis, Biomacromolecules 9,1139 (2008).

17. D. W. Van Krevelen Properties of polymers. $3^{\text {rd }}$ Ed. Amsterdam. Elsevier, 1990.

18. N. M. Neves, G. Isdell, A. S. Pouzada and P. C. Powell, Polym. Compos. 19, 640 (1998).

19. H. Mahfuz, A. Adnan, V. K. Rangari, S. Jeelani and B. Z. Jang, Composites: Part A 35, 519 (2004).

20. W. Krause, F. Henning, S. Tröster, O. Geiger and P. Eyerer, J. Thermoplas. Compo. Mater. 16, 289 (2003).

21. V. M. Correlo, L. F. Boesel, M. Bhattacharya, J. F. Mano, N. M. Neves and R. L. Reis, Mater. Sci. Eng. A 403, 57 (2005).

22. M. P. Pavlov, J. F. Mano, N. M. Neves and R. L. Reis, Macromol. Biosci. 4, 776 (2004). 